\title{
Alumnado femenino en las enseñanzas de artes plásticas y diseño en el sistema educativo público español 1940-2015
}

\author{
Female students in the teaching of fine arts and design in the \\ spanish public education system 1940-2015
}

Marta QUÍLEZ. Universidad Miguel Hernandez (España). quilezindiam@gmail.com

Resumen: El presente artículo se encarga de analizar la incorporación del alumnado femenino a la enseñanza de Artes Plásticas y Diseño dentro de un marco temporal referenciado que abarca desde el final de la Guerra Civil española hasta la actualidad. El propósito es examinar la escasa evolución de la mujer dentro del sistema educativo en una etapa marcada por la adquisición de derechos. Se pretende analizar social y cualitativamente el lugar que la mujer ocupaba y ocupa en la configuración de un sistema educativo complejo, en nuestra historia reciente. En este contexto se analizan las especialidades artísticas más demandadas por el sexo femenino en las enseñanzas de bachillerato, formación profesional y estudios superiores no universitarios.

Para ello debemos conceptualizar y situar estas enseñanzas a lo largo de la historia y cómo los cambios acontecidos durante su evolución han afectado al alumnado femenino.

Las enseñanzas de Artes Plásticas y Diseño son herederas de la tradición reformista que el Movimiento Arts \& Crafts iniciado en Europa por William Morris en el siglo XIX. Durante más de doscientos años ha evolucionado condicionadas, siempre, por la inestabilidad del cambiante sistema educativo español.

Palabras clave: Educación, mujer, estudios artísticos, escuelas de arte, artes plásticas, diseño. 


\begin{abstract}
This article is responsible for analyzing female students' incorporation to the teaching of Fine Arts and Design since the end of the Spanish Civil War to the present.
\end{abstract}

The purpose is to examine the modest evolution of women in the education system during a time marked by the acquisition of rights. It also aims to analyse socially and qualitatively the place that women occupied and occupy in the configuration of a complex education system in our recent history. In this context, the most demanded artistic specialties by the female sex are analyzed in the baccalaureate, vocational training and non-university higher education courses.

For this reason, we must conceptualize and situate these teachings throughout history and how the changes occurred during their evolution have affected female students. The teachings of Fine Arts and Design are heiresses to the reformist tradition that the Arts \& Crafts Movement initiated in Europe by William Morris in the 19th century. For more than two hundred years it has evolved, always conditioned, by the instability of the changing Spanish educational system.

Keywords: Education, women, artistic studies, art schools, plastic arts, design.

\title{
Introducción
}

El objeto de estudio de este artículo es investigar en el panorama educativo dentro de las enseñanzas de Artes Plásticas y de Diseño en España, comenzando a mediados del siglo XX hasta la actualidad, desde una perspectiva feminista como forma de reivindicación social femenina y analizar cómo la mujer se incorporó a la educación en las Escuelas de Artes y Oficios, abriendo paulatinamente los espacios públicos y profesionales que habían permanecido clausurados a su acceso.

Durante la renovación del siglo XX la mujer aprovechó la enseñanza profesional de las Artes y Oficios ayudada por la modernización industrial, dónde encontró la forma de acceder a mercado laboral y de esta manera, consiguió incipientemente salir de los límites sociales impuestos por el patriarcado que le impedían ocupar su puesto en la sociedad como ciudadana, trabajadora y mujer.

La finalidad de este trabajo de investigación tiene dos metas precisas, desde una perspectiva histórica: el primero la formación artística de las mujeres, en el estado español y un segundo en el que se analizan las matriculaciones por ramas formativas artísticas en el periodo citado.

No obstante, la mayoría de los trabajos de investigación en los que se empieza a tener constancia de la mujer como objeto de estudio surgen a partir de 1960, en su totalidad parten de un hecho social evidente: la mujer se ha ido incorporando 
progresivamente al mundo laboral, artístico y científico. Esta anexión en progresión ascendente, ha contribuido a una valoración real como persona creadora, aunque queda mucho por hacer para alcanzar la igualdad, la labor que actualmente la mujer está realizando, nos indica que todavía a día de hoy sigue habiendo impedimentos y resulta complicado conseguir una equiparación real con el sexo masculino. Todos estos esfuerzos, llevados a cabo por mujeres, tanto colectivos como los particulares, tienen su origen en la lucha de la mujer en su día a día y en la realidad social que la rodea. Todos estos esfuerzos tienen un fin determinado: conseguir una sociedad totalmente igualitaria.

Para llegar al punto actual donde nos encontramos, con un entramado complejo de opciones educativas en el campo de Artes Plásticas, es imprescindible, analizar la historia para ser conscientes de la complejidad de dichos estudios. Este tipo de enseñanzas no son universitarias y desde sus inicios hasta la promulgación de la ley de Educación de 1990 (LOGSE) no fueron completamente definidas. Aún todavía hoy existen brechas legislativas cuya solución está en manos de la administración.

Es considerable distinguir entre las enseñanzas de Música, Danza y Arte Dramático y por otra parte las enseñanzas de Artes Plásticas, Diseño y Conservación de Bienes Culturales. Este modelo de enseñanzas se define bajo el nombre de: Enseñanzas de Régimen Especial. Sobre estas últimas versa el artículo, centrándonos principalmente en las dos primeras: Artes Plásticas y Diseño. La implantación de este tipo de enseñanzas en España ha surgido y evolucionado a partir de una demanda social y laboral cambiante.

\section{De las asociaciones gremiales a la ii república}

El primer antecedente histórico de las enseñanzas de Artes Plásticas y Diseño que nos encontramos es la tradición artesana, el origen de los oficios artísticos, tanto en España como en Europa dónde florecen los gremios, aproximadamente a finales de la Edad Media, coincidiendo con un incipiente desarrollo de las sociedades urbanas, y con ellas, nace una nueva clase social que desarrolló sus actividades mediante la agrupación en gremios. Esta estructura colectiva posee una configuración ordenada de estricto cumplimiento para asegurar que el avance profesional basado en el aprendizaje y la transmisión de conocimientos adquiridos se preservarán durante siglos.

Las enseñanzas eran principalmente orales y prácticas, con carencia de conceptos y falta de contenidos experimentales, lo opuesto al de la educación normativa.

La educación gremial se estructuraba en tres niveles: primer grado o aprendiz, segundo grado u oficial, los aprendices lo alcanzaban tras 3 o 4 años de trabajo, en este nivel estaba remunerado y el tercer grado o maestría, en el que el oficial podía realizar grandes obras, siempre por debajo del Maestro de taller. 
Durante este periodo la educación únicamente masculina, rara vez se permitía el acceso a las mujeres en los gremios tradicionales, y si lo hacían, solamente se aceptaban viudas de hombres que habían pertenecido en vida a la agrupación. También existían diferentes gremios en las que sí se permitía, algunos muy feminizados, como el gremio de las bordadoras y el de las hilanderas.

El sistema económico basado en la agricultura mantiene su predominio en España, inalterado hasta principios de siglo, convive también, con una producción industrial poco desarrollada de escasa evolución técnica, esta, se sostiene gracias a la mano de obra sin cualificación de obreros de clases bajas. La formación técnica de los operarios sigue siendo la popular: gremios y asociaciones, mediante el método clásico de aprendizaje de las mismas.

Durante el siglo XVIII la ilustración fue muy crítica con el modelo gremial, les acusaban de dificultar la libre competencia. La abolición definitiva del monopolio gremial llegó en la época de la regencia de Isabel II en 1834¹, facilitando la aparición de otras entidades públicas y privadas que afrontan y desarrollan nuevos marcos para el desempeño de actividades. En aquí cuando nacen las Sociedades Económicas de Amigos del País ${ }^{2}$ que junto con las Reales Fábricas ${ }^{3}$ y Consulados cuya finalidad principal era la de dar continuidad a las enseñanzas y perfeccionamiento de las clases trabajadoras.

Gracias a los de los ministros ilustrados Jovellanos y Campomanes, junto con las Sociedades Económicas de Amigos del País y las Juntas de Comercio, crearán las "Escuelas Patrióticas de Dibujo" que emergerán por todo el país instauradas y supervisadas por ambas entidades.

También se crea la "Escuela Gratuita de Diseño" fundada en 1774 en Bilbao, y un año más tarde en Barcelona, la "Casa de la Lonja del Mar", sufragada por la Junta de Comercio de la ciudad, tomando esta como referencia, se van instaurando diferentes escuelas por toda España.

En 1871, se implantan las Escuelas de Artes y Oficios, creándose la primera en Madrid como un anexo del Conservatorio Superior. De esta forma, se equiparaba la

1. Real Decreto 20 de enero de 1834. Regulación de los gremios y la libertad de fabricación.

2. A partir de la segunda mitad del siglo XVIII, se fundaron varias Sociedades Económicas de Amigos del País, organismos no estatales, con el fin de promover el desarrollo de España, especialmente en el aspecto económico, y que se inició en los círculos culturales. Contaban con licencia real para constituirse y reunirse, y en su fundación intervinieron los sectores más dinámicos de la sociedad.

3. Ley Moyano de Instrucción Pública de 1857. Instalaciones industriales que se fundan por iniciativa de los monarcas absolutos durante el Antiguo Régimen, como resultado de la aplicación de la política mercantilista. Muchas de ellas se dedicaban a la fabricación de objetos de lujo, para el consumo en primer lugar de los propios palacios reales. 
educación artística con la técnica, tendencia educativa de la época en ese momento. En 1870 nacía la primera Escuela de Artes y Oficios, independiente. El Real Decreto de 1886 facilita la creación de siete escuelas más en territorio español.

La incorporación de la mujer en este sistema educativo fue un proceso lento y largo, por primera vez en 1857 tenían derecho a una educación formal, gracias a la Ley de instrucción pública del 9 de septiembre de $1857^{4}$. La ley establecía la obligatoriedad de la enseñanza primaria y publica para hombres y mujeres, así como la creación de centros educativos diferenciados por sexo. Las materias también fueron diferentes segmentando por sexo, dejando a las mujeres en la educación primaria las labores domésticas, dibujo e higiene en el hogar, en contraste con los hombres que cursaban, agricultura, comercio, industria, historia y física y química.

El nacimiento de las Escuelas de Artes y Oficios tiene un objetivo muy concreto, aportar un nivel cultural básico a las clases sociales medias y bajas. El tipo de educación que se impartía en ellas era de carácter técnico y las materias que se enseñaban estaban enfocadas a la búsqueda y desempeño de trabajo a corto plazo, esta preparación estaba en consonancia con las necesidades en las que el país se encontraba en ese momento, abastecer a una pequeña burguesía industrial.

La industrialización, como dice Geraldine M. Scanlon, donde mayor efecto causó fue en las vidas de las mujeres de clase baja, contribuyendo a la modificación de los planes de educación orientados hacia la mujer de clase media ${ }^{5}$. Esta primera aceptación de la mujer en un pequeño sector del mundo profesional, incluía la necesidad de una escolarización básica en los niveles de enseñanza elementales, pero principalmente en los niveles superiores prometiendo un futuro puesto laboral que mejoraría su posición, a la vez que una liberalización social.

Esta última afirmación da un nuevo enfoque a la clásica interpretación histórica en la que el proceso de industrialización ser origina en las clases obreras y medias a modo de desahogo a la economía familiar, y como un futuro prometedor más estable para las clases medias.

No solamente debemos incluir en este discurso que fueron las necesidades económicas y materiales lo que llevó a las mujeres de clases medias a buscar un empleo remunerado, hemos de tener en cuenta la vocación profesional, siempre en el caso de la mujer condicionada a las relaciones de poder que implicaba su género, toda mujer podía estudiar en una escuela profesional siempre y cuando los estudios

\footnotetext{
4. Ley Moyano de Instrucción Pública de 1857.

5. Planteamiento en la obra de SCANLON, Géraldine M.: La polémica feminista en la España contemporánea (1868-1974). Madrid, Siglo XXI, 1976
} 
que realizará estuvieran enmarcados dentro de los límites del carácter de su propio sexo.

La primera reorganización oficial de la enseñanza femenina en las Artes y Oficios fue en el año 1895. En la disposición aparecen cuatro secciones de estudio para la Escuela Central de Madrid: los siete grupos preparatorios, la sección técnicoindustrial, una sección artístico-industria y la enseñanza artístico-industrial de la mujer, compuesta por las materias: período preparatorio con Aritmética y geometría, Dibujo lineal, Dibujo de adorno y figura. En el período de ampliación: Dibujo de adorno y figura y Elementos de colorido, Modelado y vaciado de adorno y figura. En cuanto a las enseñanzas prácticas femeninas, fueron: Confección de flores, Bordados, Encajes y Tapicería.

Queda demostrado que la presencia femenina en esta escuela existía con anterioridad, en el que las mujeres accedían a la educación mediante la matrícula libre. ${ }^{6}$

Además, otras escuelas de provincia aseguran la presencia de alumnas ya desde la década de los ochenta del siglo XIX. Referencias que probarían la existencia de una conciencia femenina que confiaría en el camino de la formación profesional como vía para la promoción socioeconómica. Por ello, la exigencia y el contexto social es el que fuerza a la ley a la inclusión de la mujer dentro de la legislación oficial de la formación profesional.

Durante esta época los tres modelos de enseñanza artística: Escuelas Patrióticas de Dibujo, Escuelas Gratuitas de Dibujo y las surgidas bajo la tutela del Real Conservatorio de Artes confluirán tras la promulgación de un Real Decreto en 1900 creándose las Escuelas de Artes e Industrias reorganizando y uniendo las Escuelas de Artes y Oficios y las provinciales de Bellas Artes, en dos secciones: técnico industrial y artístico industrial, solo duró hasta 1910 cuando se desligaron definitivamente los sectores artísticos y técnicos.

Los avances en el resto de ramas educativas femeninas a partir de 1915 fueron importantes en consiguiendo crear varios centros de Institutos-escuela primarios y secundarios mixtos y la inauguración de la primera institución oficial con carácter universitario para mujeres graduadas.

La proclamación de la II República, el 14 abril de 1931, supuso un gran progreso en toda la educación española, estableció una política educativa basada en de escuela única, pública, laica y gratuita. La Constitución de 1931 trajo la igualdad para las mujeres, reconociendo igualdad géneros en la educación y en el ámbito laboral.

6. Reglamento del 3 de junio de 1873, núm. 156, p. 628 (Gaceta del 5) y R.D. del 5 de noviembre de 1886, núm. 310, p. 378 (Gaceta del 6) 


\section{La educación en el largo régimen franquista}

Durante la dictadura franquista la educación estuvo bajo las ordenes de la Falange y la Iglesia, esta, consiguió vía libre para crear centros educativos ligados a órdenes religiosas, de esta manera se conseguían contenidos educativos catolizados.

La Falange, por su parte se ocupó de "completar la educación de las mujeres" a través de la Sección Femenina, y esta fue la responsable de volver a formar a las mujeres en las enseñanzas propias de su género. En las Escuelas Hogar de Artes Sindical, las alumnas asistían en horario vespertino, para poder compatibilizar con su jornada laboral, recibiendo clases prácticas y teóricas. Las asignaturas prácticas estaban compuestas por: labores, corte y confección manualidades, telares y cocina. Las asignaturas teóricas incluían economía doméstica, religión, puericultura, formación familiar y social, música e historia. ${ }^{7}$

Tabla 1.

Matriculación alumnado total y por sexo 1940 - 2016

\begin{tabular}{ccccccc} 
& TOTAL & MUJERES & HOMBRES & MUJERES \% & HOMBRES \% \\
\hline $\mathbf{1 9 4 0 - 1 9 5 0}$ & $\mathbf{2 1 . 8 9 8}$ & $\mathbf{6 . 4 1 6}$ & 15.460 & $29,30 \%$ & $70,60 \%$ \\
$\mathbf{1 9 5 1 - 1 9 6 0}$ & $\mathbf{1 9 . 5 5 9}$ & $\mathbf{6 . 9 4 3}$ & 12.596 & $35,50 \%$ & $64,40 \%$ \\
$\mathbf{1 9 6 1 - 1 9 7 0}$ & $\mathbf{1 8 . 5 7 3}$ & $\mathbf{8 . 3 0 2}$ & 10.252 & $44,70 \%$ & $55,20 \%$ \\
$\mathbf{1 9 7 1 - 1 9 8 0}$ & $\mathbf{3 4 . 3 4 2}$ & $\mathbf{2 0 . 3 9 9}$ & 13.909 & $59,40 \%$ & $40,50 \%$ \\
$\mathbf{1 9 8 1 - 1 9 9 0}$ & $\mathbf{2 6 . 1 6 8}$ & $\mathbf{1 5 . 7 7 9}$ & 10.363 & $60,30 \%$ & $39,60 \%$ \\
$\mathbf{1 9 9 1 - 2 0 0 0}$ & $\mathbf{2 2 . 0 6 5}$ & $\mathbf{1 3 . 8 1 3}$ & 8.230 & $62,60 \%$ & $37,30 \%$ \\
$\mathbf{2 0 0 1 - 2 0 1 0}$ & $\mathbf{2 3 . 2 1 1}$ & $\mathbf{1 4 . 2 9 8}$ & 8.913 & $61,60 \%$ & $38,40 \%$ \\
$\mathbf{2 0 1 1 - 2 0 1 6}$ & $\mathbf{2 8 . 6 9 3}$ & $\mathbf{1 7 . 5 6 0}$ & 11.104 & $61,20 \%$ & $38,70 \%$
\end{tabular}

Nota. Gráfico de elaboración propia Fuente: Anuarios Estadísticos 1940-1969, Instituto Nacional de Estadística 1970-1985, Ministerio de Educación 1986- 2017.

En cuanto al análisis estadístico de la educación en la década de 1940 la matriculación femenina en las Escuelas de Artes y Oficios era bastante inferior en relación al número de alumnos masculinos, las mujeres solamente representaban el 29,30\% del global del alumnado registrado (Tabla 1).

7. Memoria de la labor realizada por en la Escuela Hogar de Artes sindical de la Sección Femenina de Teruel. Curso 19561957. Fuente: Archivo Histórico Provincial de Teruel. 


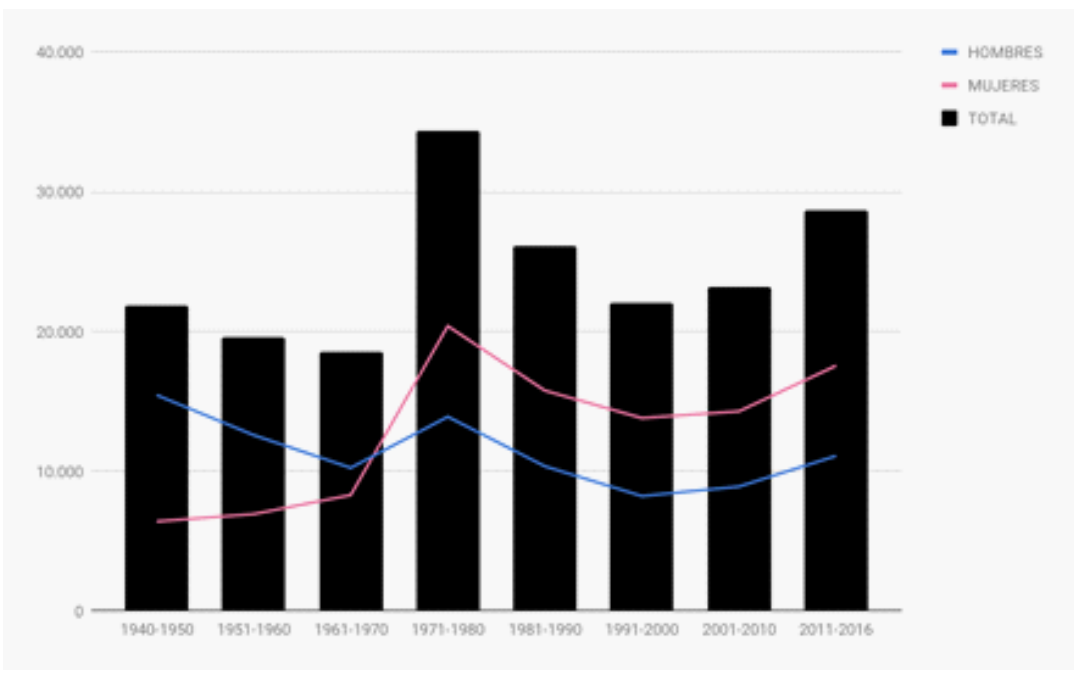

Figura1.Evolucióndelamatriculaciónfemeninapordécadas 1940-2016. Gráfico de elaboración propia. Fuente: Anuarios Estadísticos 1940-1969, Instituto Nacional de Estadística 1970-1985, Ministerio de Educación 1986- 2017.

En referencia a la Figura 1, se puede observar a priori la matriculación global de ambos sexos fue ascendente hasta la década de los 70 y 80 para posteriormente descender y repuntar hasta la actualidad, en general, desde esta década hasta la actualidad la matriculación por género se ha mantenido estable.

Durante la década de los años 50 la situación social y económica del país mejoró notablemente. En las escuelas de Escuelas de Artes y Oficios la matriculación de alumnas durante esta década fue superior teniendo una media de 500 alumnas más, inscritas por curso, siendo la matriculación femenina un $35,50 \%$ del total de matrículas durante los años 50. La década de los 60, viene marcada por el aperturismo social y político de España, este, exigía un cambio estructural del sistema educativo que lo adaptase al presente. Es una década de grandes avances en la educación femenina, en las universidades la mujer ya representaba el $26 \%$ y en la educación primaria igualaba por primera vez al número de alumnos matriculados. En las de Escuelas de Artes y Oficios la matricula femenina aumentó casi un 10\% respecto a la década anterior, con 2.000 mujeres más de media en cada curso escolar, es en esta década cuando por primera vez en la mujer consigue superar en número de matriculaciones al hombre, lo hace en el curso escolar $67 / 68$ siendo un 50,47\%.

Es en 1963, con el Decreto 2127/1963, de 24 de junio ${ }^{8}$, cuando comienza a legislar el reglamento de las Escuelas de Artes Aplicadas y Oficios Artísticos, mediante el decreto se estableció una normativa que supuso el reconocimiento de nuevas profesiones especializadas: Diseño, Arte Publicitario y Decoración; con ello se

8. Decreto 2127/1963, de 24 de julio, sobre reglamentación de los estudios de las Escuelas de Artes y Oficios Artísticos. Boletín Oficial del Estado núm. 214, de 6 de septiembre de 1963, páginas 13088 a 13090 
pretendía dar un giro a estas enseñanzas y dejar atrás su carácter artesanal para poder ofrecer una respuesta educativa a los nuevos tiempos y más acorde la demanda de la sociedad del momento.

De esta manera, las escuelas pasan a llamarse: "Escuelas de Artes Aplicadas y Oficios Artísticos" y se crean 4 secciones: Decoración y Arte Publicitario, Diseño, delineación y trazado artístico, Artes aplicadas al libro y Taller de Artes Aplicadas, dentro de ellas se englobaban diversas especialidades. Se crea un currículo con enseñanzas distribuidas en 5 cursos lectivos, de los cuales los tres primeros poseen las asignaturas comunes y los 2 últimos tienen asignaturas propias de la especialidad.

\section{El gran salto en 1970 de la ley general de educación}

El gran cambio educativo vino en 1970 con la promulgación de la Ley General de Educación (LGE) ${ }^{9}$ de Villar Palasí, se avanzaba dejando atrás el marco legal que regía el sistema educativo, que aún estaba administrado, un siglo después, por la Ley Moyano.

Uno de los grandes hitos a destacar en esta década es que por primera vez la mujer supera al hombre en matriculaciones, estos logros se consiguen gracias a la creación de escuelas e institutos mixtos que ofrecía por primera vez, desde la II República un currículo sin división por sexo.

Para las Escuelas de Artes, la LGE supuso todavía más cambios, distribuyó la educación artística en dos niveles, uno superior en el que las enseñanzas artísticas adquirían la categoría de Escuela Universitaria y otro básico, el de la enseñanza profesional. El primer punto no se desarrolló, poniéndose en práctica en el año 1992. Desde otro punto de vista, los primeros pasos para la reforma del sistema educativo, van a favorecer la ampliación de las enseñanzas introduciendo nuevos cursos comunes y nuevas especialidades "experimentales", entre las que aparecen la de Diseño Industrial en 1984, Diseño de Textiles y Moda, Diseño Gráfico, Diseño de Interiores y Diseño de Orfebrería y Joyería, en 1987.

Con relación a registros, la femenina fue un $10 \%$ superior a la masculina en el cómputo global de las enseñanzas. A partir de esta década se obtienen datos más precisos sobre matriculaciones y sección, que comenzaremos a analizar en la Figura 2, la sección con mayor número de matriculaciones es: Decoración y Arte Publicitario, dónde las mujeres representan un $61,21 \%$ del total.

9. Ley 14/1970, de 4 de agosto, General de Educación y Financiamiento de la Reforma Educativa. Boletín Oficial del Estado núm. 187, de 6 de agosto de 1970, páginas 12525 a 12546 
De las cuatro secciones solamente en dos los hombres superan en matriculaciones es en: Diseño, Delineación y Trazado Artístico, una de las ramas de estudio más técnicas y profesionalmente más masculinizada y Artes aplicadas al Libro, área relacionada con el mundo de la impresión y artes gráficas, sector tradicionalmente dominado por hombres.

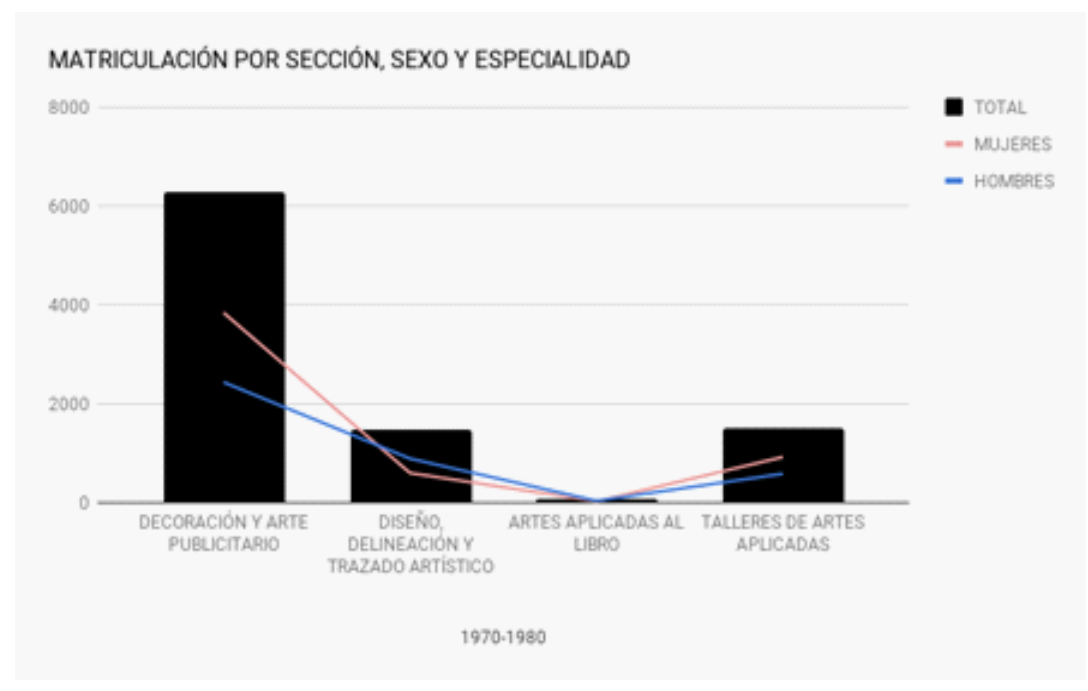

Figura 2. Matriculación alumnado por sección y sexo 1970 - 1980. Gráfico de elaboración propia. Fuente: INE.

Tras la muerte de Franco en 1975 llegaron las primeras elecciones democráticas desde 1936, el nuevo parlamento diseña una constitución democrática, el artículo 27 marcó los principios generales de toda la legislación actual en materia educativa, introduciendo, como es lógico, profundas diferencias de enfoque y modificaciones parciales.

El ajuste de los principios democráticos y de participación presente en la Constitución impulsa la aprobación de nuevas leyes educativas. Será el gobierno del Partido Socialista Obrero Español, quien se encargue de dicha tarea, aprobando cuatro leyes orgánicas básicas que, junto con la Constitución, regulan el sistema educativo español. 
QUÍLEZ / Alumnado femenino en las enseñanzas de artes plásticas y diseño en el sistema educativo público español 1940-2015

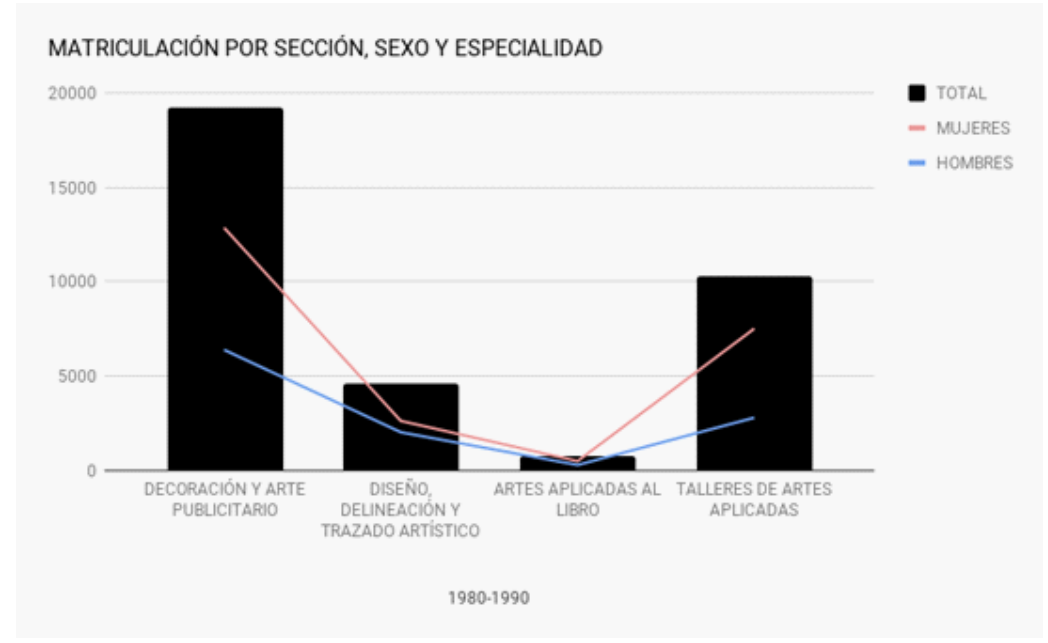

Figura 3. Matriculación alumnado por sección y sexo 1980 - 1990. Gráfico de elaboración propia. Fuente: INE.

Tabla 2

Matriculación alumnado por sección y sexo 1980 - 1990

MATRICULACIÓN POR SECCIÓN 1980-1990

\begin{tabular}{lc|c|c|c} 
& TOTAL & MUJERES & HOMBRES & MUJERES \% \\
\hline $\begin{array}{l}\text { DECORACIÓN Y ARTE } \\
\text { PUBLICITARIO }\end{array}$ & $\mathbf{1 9 2 4 5}$ & 12859 & 6386 & $66,82 \%$ \\
$\begin{array}{l}\text { DISEÑO, DELINEACIÓN Y } \\
\text { TRAZADO ARTÍSTICO }\end{array}$ & $\mathbf{4 6 2 9}$ & 2615 & 2014 & $56,49 \%$ \\
$\begin{array}{l}\text { ARTES APLICADAS AL LIBRO } \\
\text { TALLERES DE ARTES }\end{array}$ & $\mathbf{7 6 3}$ & 485 & 278 & $63,56 \%$ \\
APLICADAS & $\mathbf{1 0 2 9 9}$ & 7512 & 2787 & $72,94 \%$ \\
\hline TOTAL & $\mathbf{3 4 9 3 6}$ & 23471 & 11465 & \\
\hline & & $67,18 \%$ & $48,85 \%$ &
\end{tabular}

Nota. Gráfico de elaboración propia. Fuente: INE.

Durante la década de los 80 se consolidaron los cambios de ley realizados en la década anterior, comienzan varios planes que incluyen estudios experimentales, como el bachillerato artístico, que se desarrollaría posteriormente.

En lo referente a matriculación general las escuelas experimentaron un fuerte aumento de 7.892 a 34.936 de alumnos con respecto a la década anterior. En la matriculación desglosada por secciones puede comprobarse que en todas las secciones las mujeres superan a los hombres, incluso en Talleres de Artes Aplicadas alcanza un 72,94\%, en las Artes Aplicadas al Libro, tradicionalmente masculina la cifra alcanzó un 20\% más de mujeres en las aulas, por contrapunto la especialidad menos demandada por 
el sector femenino sigue siendo Diseño Trazado y Delineación Artística, aun así, las mujeres son un $56,49 \%$.

\section{LOGSE. Acabando lo empezado}

La promulgación de la LOGSE establece un marco único para las enseñanzas artísticas, las sitúa dentro de las llamadas “Enseñanzas de Régimen Especial”, estas, se dividieron una vez más en tres secciones: música y danza, arte dramático y artes plásticas y diseño. Así mismo se crean dos niveles diferentes, el primero de ellos los estudios Superiores que no se implantaron hasta el final de la década y los grados de formación profesional: medio y superior, que incluían al finalizar la formación en empresas y completando así el acceso al mundo laboral. ${ }^{10}$

Otra de las medidas de organización del sistema educativo de la LOGSE llegó en 1992, cuando mediante un Real Decreto se implantó definitivamente por primera vez en España una rama de bachillerato de específica de artes, esta implantación conlleva un paso adelante que supone una consolidación de las enseñanzas artísticas en un sistema educativo en el que siempre se las ha obviado. Al igual que en el resto de especialidades artísticas la matriculación femenina en ambos cursos de Bachillerato artístico a lo largo de la historia desde su implantación definitiva, es superior en un $63,75 \%$ de media respecto a la matrícula masculina, llegando a ser un punto superior en segundo curso (Tabla 3).

Tabla 3

Matriculación alumnado por sección y sexo 1980-1990

MATRICULACIÓN BACHILLERATO ARTES PLÁSTICAS CURSOS: 1994-2015

\begin{tabular}{|c|c|c|c|c|}
\hline & TOTAL & MUJERES & HOMBRES & MUJERES \% \\
\hline $1^{\circ}$ CURSO & 219988 & 138682 & 81306 & $63,04 \%$ \\
\hline $2^{\circ}$ CURSO & 202782 & 130817 & 71965 & $64,51 \%$ \\
\hline TOTAL & 422770 & & & \\
\hline & & $63,75 \%$ & $36,25 \%$ & \\
\hline
\end{tabular}

Nota. Gráfico de elaboración propia. Fuente: INE.

10. Artículo 48. Ley Orgánica 1/1990, de 3 de octubre, de Ordenación General del Sistema Educativo 
Con la nueva distribución curricular de la LOGSE se crean diferentes familias profesionales que se ocupan de las enseñanzas de Grado Medio y dan continuidad con las de Grado Superior. Durante la década de los 90 conviven planes de enseñanza antiguos y actuales, no es hasta 1996 cuando se comienza establecer los diferentes currículos de las familias educativas, quedando definitivamente implantados en 1998.

Tabla 4

Matriculación alumnado Grado Medio, familia y sexo 1995 - 2015

CICLOS FORMATIVOS GRADO MEDIO: 1995-2015

\begin{tabular}{l|cccc}
\hline & TOTAL & MUJERES & HOMBRES & MUJERES \% \\
\cline { 2 - 5 } ESCULTURA & $\mathbf{1 7 1 4 0}$ & 11315 & 5825 & $33,98 \%$ \\
INDUMENTARIA & $\mathbf{8 4 2}$ & 172 & 670 & $79,57 \%$ \\
LIBRO & $\mathbf{3 1 8 9}$ & 1513 & 1676 & $52,56 \%$ \\
CERÁMICA & $\mathbf{5 5 4 4}$ & 1808 & 3736 & $67,39 \%$ \\
DISEÑO GRÁFICO & $\mathbf{1 5 5 5 1}$ & 8743 & 6808 & $43,78 \%$ \\
JOYERÍA & $\mathbf{2 8 1 6}$ & 1025 & 1791 & $63,60 \%$ \\
TOTAL & $\mathbf{4 5 0 8 2}$ & 20.506 & 24.576 & \\
\hline & & $45,49 \%$ & $54,51 \%$ &
\end{tabular}

Nota. Gráfico de elaboración propia. Fuente: INE.

El análisis sigue el mismo flujo estable ambos ciclos. Hay enseñanzas que tienen una matrícula superior de mujeres y otros, de tradición masculina tienen un porcentaje ínfimo de alumnas, así que existen ramas exclusivamente de mujeres en grado medio, como son cerámica e indumentaria y en grado superior como: cerámica, diseño de interiores o joyería.

Por el contrario, los ciclos de la familia de escultura siempre han tenido un alto grado de registro masculino, así mismo hay familias en las que la matriculación en grado medio, como diseño gráfico el número de hombres es superior, pero en las enseñanzas de grado superior no. En grado medio solamente hay dos familias en la que la matriculación de mujeres es inferior, y es la de artes aplicadas a la escultura, y diseño industrial el resto de ramas la matrícula femenina es superior, ajustada en algunos casos como es el del diseño gráfico y Artes Aplicadas al Libro, otras, sin embargo, como Joyería Artística o Diseño de Interiores, alcanzan cifras del 80\% matriculación de alumnas (Tabla 4). 
Tabla 5

Matriculación alumno, especialidad y sexo

CICLOS FORMATIVOS GRADO SUPERIOR: 1995-2015

\begin{tabular}{l|cccc}
\hline & TOTAL & MUJERES & HOMBRES & MUJERES \% \\
\cline { 2 - 3 } ESCULTURA & $\mathbf{1 6 6 5 2}$ & 7665 & 8987 & $46,03 \%$ \\
LIBRO & $\mathbf{5 2 8 6}$ & 2966 & 2320 & $56,11 \%$ \\
CERÁMICA & $\mathbf{1 0 7 7 9}$ & 7303 & 3476 & $67,75 \%$ \\
DISEÑO GRÁFICO & $\mathbf{1 0 2 7 3 4}$ & 54988 & 47746 & $53,52 \%$ \\
D. INDUSTRIAL & $\mathbf{8 2 2 5}$ & 3390 & 4835 & $41,22 \%$ \\
D. INTERIORES & $\mathbf{4 9 2 2 4}$ & 40122 & 9102 & $81,51 \%$ \\
JOYERÍA & $\mathbf{7 7 2 0}$ & 6281 & 1439 & $81,36 \%$ \\
TOTAL & $\mathbf{2 0 0 6 2 0}$ & $\mathbf{1 2 2 7 1 5}$ & $\mathbf{7 7 9 0 5}$ & \\
\hline & & $61,17 \%$ & 38,83 &
\end{tabular}

Nota. Gráfico de elaboración propia. Fuente: Estadística de las Enseñanzas no universitarias. Subdirección General de Estadística y Estudios del Ministerio de Educación, Cultura y Deporte.

En los estudios de Grado Superior nos encontramos con escenario similar al visto en la formación inferior, ciclos completamente feminizados como Joyería y Diseño de Interiores, ciclos equilibrados como Diseño Gráfico y Artes Aplicadas al Libro. Al contrario, tenemos un nuevo ciclo como es el de Diseño Industrial, en el que las mujeres son minoría un $41,22 \%$, lo cursa, así mismo el ciclo que de Escultura, que da continuidad al grado medio, manifiesta el mismo patrón. (Tabla 5).

\section{Subiendo otro escalon: estudios superiores}

La publicación de la LOGSE en 1990, ampliaba el horizonte de las enseñanzas de artes plásticas y diseño, por un lado, definió y separó las Escuelas Superiores de Artes Plásticas y las Escuelas Superiores de Diseño, que impartirían los Estudios Superiores de Artes Plásticas y de Diseño, correspondientemente, y las Escuelas de Arte, que continuaban con los Ciclos Formativos de Artes Plásticas y Diseño; pero no fue hasta $1999^{11}$ que se establecieron los planes de estudio de los Estudios Superiores de Diseño, equivalentes a diplomatura universitaria, aunque su ubicación dentro del organigrama de las distintas administraciones educativas no resultó acorde con el nivel de los estudios, seguían estando dentro de las enseñanzas de régimen especial, gestionadas y tratadas como educación secundaria no obligatoria. El plan Bolonia sentó las bases para empezar un proceso de convergencia de los sistemas educativos de los niveles superiores de la enseñanza.

11. REAL DECRETO 1496/1999, de 24 de septiembre, por el que se establecen los estudios superiores de Diseño, la prueba de acceso y los aspectos básicos del currículo de dichos estudios. BOE 6/10/1999 
La Ley Orgánica 2/2006, de 3 de mayo ${ }^{12}$, de Educación ubicó a las Enseñanzas Artísticas Superiores en un espacio propio y flexible dentro del marco de la Educación Superior Europea. Esta ley, compone los estudios superiores de diseño los tres ciclos: Grado, Máster y Doctorado, este último en convenio con la universidad. Actualmente más de 10 años después el segundo ciclo de los estudios, el nivel de Máster no se ha desarrollado al completo, pocas escuelas actualmente ofrecen programas especializados para cada especialidad, los programas de doctorado siguen sin salir adelante por falta de convenios con la universidad.

Tabla 7

Matriculación alumno, especialidad y sexo

ESTUDIOS SUPERIORES DISEÑO CURSOS: 2000-2015

\begin{tabular}{lllll}
\hline & TOTAL & MUJERES & HOMBRES & MUJERES \% \\
GRÁFICO & $\mathbf{2 4 2 6 4 5}$ & 12743 & 11902 & $51,71 \%$ \\
PRODUCTO & $\mathbf{9 4 5 1}$ & 4859 & 4592 & $51,41 \%$ \\
INTERIORES & $\mathbf{2 0 9 5 2}$ & 16264 & 4688 & $77,63 \%$ \\
MODA & $\mathbf{1 1 7 7 7}$ & 10166 & 1611 & $86,32 \%$ \\
TOTAL & $\mathbf{6 0 6 4 5}$ & 39972 & 20673 & \\
& & $65,91 \%$ & $34,09 \%$ &
\end{tabular}

Nota. Gráfico de elaboración propia. Fuente: Estadística de las Enseñanzas no universitarias. Subdirección General de Estadística y Estudios del Ministerio de Educación, Cultura y Deporte.

Si analizamos los datos recogidos sobre la matriculación del alumnado desde la implantación de los Estudios Superiores en sus cuatro familias, se puede observar como la tendencia es significativamente similar a la de los grados superiores, en general las mujeres suponen la mayoría de matrículas de todas las familias un $65,91 \%$, habiendo dos familias muy igualadas, como son la de gráfico y producto, aun así, las mujeres son superiores por un $1 \%$ en ambas. Igualmente, las carreras de interiores y moda albergan el mayor número de alumnas matriculadas, ocupaciones tradicionalmente feminizadas, mientras que los hombres optan a especialidades más técnicas.

Estas Escuelas, a pesar de sus distintas denominaciones, estructuras y ubicaciones han enfocado su actividad docente hacia la formación del diseñador profesional, sin género siempre dentro de procesos de continua experimentación y actualización según los requerimientos sociales de cada momento. Aun así, como hemos analizado a lo largo del artículo los estereotipos de género siguen influyendo en la elección de

12. Ley Orgánica 2/2006, de 3 de mayo, de Educación. Boletín Oficial del Estado núm. 106, de 04/05/2006. 
los estudios, si bien desde la etapa aperturista de la transición hasta la actualidad, los cambios en cuanto a matriculación han sido escasos, tanto en el porcentaje de alumnas como en la elección de las familias profesionales, no obstante, la interiorización de estos estereotipos tiene consecuencias educativas y sociales muy importantes, ya que constituyen un papel básico en las formas de pensar, actuar e interpretar de las personas. En el caso de las enseñanzas artísticas se confirma, una vez más, las ramas más feminizadas son diseño de moda, tradicionalmente ligada a la mujer y diseño de interiores, tratada a menudo como una disciplina, auxiliar de la arquitectura en la que las mujeres suelen estar alejadas de la toma de decisiones, ya que en ambas se materializa una extensión de los roles de género asignados a las mujeres, la parte estética y sensible de adornar espacios que previamente han sido creados por el hombre.

A su vez las disciplinas de diseño de producto, gráfico son las más masculinizadas, quizás por englobar conocimientos científicos y tecnológicos en su currículo o por unir la innovación y tecnología, áreas tradicionalmente más masculinas. En cualquier caso, es destacable que posteriormente en el ámbito laboral, los puestos de dirección y la toma de decisiones la presencia de las mujeres es baja, hecho que choca significativamente con el número de alumnas matriculadas.

En definitiva, si observamos cómo se toman las decisiones de formación educativa y cómo influyen los resultados y consecuencias en ambos sexos, se puede ver que se ha avanzado muy poco o nada en la superación de los estereotipos que dan lugar a la segregación formativa, y posteriormente laboral, esto supone un gran escollo en la participación democrática de hombres y mujeres en la sociedad. De dicha segregación formativa podemos afirmar que es fruto del proceso de socialización diferencial de género, que se continúa repitiendo en las aulas desde la educación primaria a la no obligatoria o universitaria. Esta misma tendencia de participación diferencial de alumnos y alumnas tendrá una importante repercusión en el futuro profesional ya que se repite a lo largo de todos los niveles educativos, siendo la rama de artes tradicionalmente una de las más feminizadas.

\section{Referencias bibliográficas}

Cabrera Rodríguez. L.J. (1997). La FP en España antes de la Ley General De Educación de 1970. Revista de educación, 312, 173-190.

Grana, I. (2002). La historia de la educación de las mujeres en España: Líneas actuales de investigación. Revista de Educación, 334, 131-141.

Grañeras, M. (2009). Las Mujeres en el sistema educativo II. Madrid: IFIIE.

Grañeras, M. e Isidoro, P. (2008). Revisión bibliográfica sobre mujeres y educación en España (1983-2007). Madrid: Instituto de la Mujer. 
QUÍLEZ / Alumnado femenino en las enseñanzas de artes plásticas y diseño en el sistema educativo público español 1940-2015

Herrero, J. y López-Salazar Pérez, C. (2011). Cien años de la Escuela de Artes de Ciudad Real 1911-2011. Ciudad Real: Diputación Provincial de Ciudad Real, BAM.

Instituto Asturiano de la Mujer. (2007). Programa de orientación académica y profesional. Oviedo: Agrupación de desarrollo Calíope.

Labarge, M. yTerán, N. (2003). La mujer en la Edad Media. Madrid: Nerea.

López, J., Vico Nieto, M., Alonso López, M., León García, A., López Crevillén, E., Martí González, M., Martínez Díaz, M., Mestre Massot, I. y Veintimilla, A. (n.d.). Las enseñanzas artísticas superiores en el Espacio Europeo de Educación Superior.

Lorente, B. (2004) Género, ciencia y trabajo. Las profesiones feminizadas y las prácticas de cuidado y ayuda social. Scripta Ethnologica, 26, 34-53.

Martín, R. (1990). La mujer como creadora: La Escuela De Artes y Oficios de Vitoria (1900-1990). KOBIE (Serie Bellas Artes), 8, 25-21.

Martínez, S. (2006). Reflexiones entre economistas y políticos sobre la enseñanza técnica la reorganización de las escuelas de artes y oficios en España (1885-1886). Revista de educación, 341, 619-642.

Ministerio de Educación y Ciencia. (1989). Libro blanco para la reforma del sistema educativo. Madrid: Centro de publicaciones

Ministerio de Educación y Ciencia. (2000). Enseñanzas artísticas. Disposiciones vigentes: Leyes orgánicas, reales decretos comunes a todas las enseñanzas artísticas. Madrid: Secretaría General Técnica.

Ministerio de Educación y Ciencia. (2010) Informe anual sobre el estado y situación de las Enseñanzas Artísticas. Madrid: Secretaría General Técnica.

Ministerio de Educación y Ciencia. (2010) Las enseñanzas artísticas superiores en el espacio Europeo de educación superior. Madrid: Secretaría General Técnica.

Montero, A.M. (1998). Origen y desarrollo de las escuelas de artes y oficios en España. Historia de la educación: Revista interuniversitaria, 17, 319-330.

Moya, F. (2012). Mujeres y trabajo en la Zaragoza del siglo XVIII. Zaragoza: Prensas Universitarias de Zaragoza.

Palanca, M.E., Navarro, P, Moral, M. (2012). 125 aniversario Escuela de Arte de Almería. Almería: Asociación de Antiguos Alumnos de la Escuela de Arte de Almería.

Rico, M.L. (2010). Mujer, Enseñanza Profesional y modernización en España (1880-1930). Historia contemporánea, 41, 447-480. 
Rico, M.L. (2016). La mujer y las Escuelas de Artes y Oficios en la España de la Restauración. Cuadernos Koré, 6, 83-113.

Sabio, B. (2006). Las Escuelas de Arte a través de la historia. Paperback. ISSN 1885-8007. 1, (1-10)

Sánchez, L. y Hernández, J. (2012). La educación femenina en el sistema educativo español (1857-2007). El Futuro del Pasado, 3, 255-281.

Satué, E. (2012). El diseño gráfico. Desde los orígenes hasta nuestros días. Madrid: Alianza Editorial

Vieites, M. F. (2016) Las enseñanzas artísticas superiores y el Espacio Europeo de Educación Superior en España. Una lectura crítica. Revista Complutense de Educación, 27(2), 499-516. 